\title{
Adenovirus mediated p53 tumour suppressor gene therapy for human gastric cancer cells in vitro and in vivo
}

M Ohashi, F Kanai, H Ueno, T Tanaka, K Tateishi, T Kawakami, Y Koike, T Ikenoue, Y Shiratori, H Hamada, M Omata

\begin{abstract}
BackgroundlAims-Gastric cancer is one of the most prevalent forms of cancer in East Asia. Point mutation of the p53 gene has been reported in more than $60 \%$ of cases of gastric cancer and can lead to genetic instability and uncontrolled cell proliferation. The purpose of this investigation was to evaluate the potential of p53 gene therapy for gastric cancer.
\end{abstract}

Methods-The responses of human gastric cancer cell lines, MKN1, MKN7, MKN28, MKN45, and TMK-1, to recombinant adenoviruses encoding wild type p53 (AdCAp53) were analysed in vitro. The efficacy of the AdCAp53 treatment for MKN1 and MKN45 subcutaneous tumours in nude mice was assessed in vivo.

Results-p53-specific growth inhibition was observed in vitro in two of four gastric cancer cell lines with mutated p53, but not in the wild type p53 cell line. The mechanism of the killing of gastric cancer cells by AdCAp53 was found, by flow cytometric analysis and detection of DNA fragmentation, to be apoptosis. In vivo studies showed that the growth of subcutaneous tumours of p53 mutant MKN1 cells was significantly inhibited by direct injection of AdCAp53, but no significant growth inhibition was detected in the growth of p53 wild type MKN45 tumours.

Conclusions-Adenovirus mediated reintroduction of wild type p53 is a potential clinical utility in gene therapy for gastric cancers.

(Gut 1999;44:366-371)

Keywords: gastric cancer; p53; adenovirus; gene therapy

Gastric cancer is one of the most common malignant tumours in the world, especially in Eastern Asia. Although the disease in the early stages is treatable by surgical resection, advanced gastric cancer does not generally respond to conventional chemotherapy or radiotherapy. ${ }^{1}$ Therefore gene therapy represents an attractive alternative for the treatment of gastric cancer. The importance of developing such therapeutic strategies is emphasised by the fact that patient survival has improved only marginally in the past two decades. ${ }^{2}$ Recent advances in molecular genetics have revealed the functional loss of the p53 gene as the most common event in carcinogenesis. ${ }^{3}$ In its role as a "guardian of the genome", p53 participates in several pathways of the cell cycle, including (a) activation of genes that inhibit cell cycle progression into the $S$ phase, (b) promotion of DNA repair, and (c) induction of apoptosis. ${ }^{4}$ Point mutation of the p53 gene has been reported in more than $60 \%$ of gastric cancers irrespective of the histological type, and can lead to the genetic instability and uncontrolled cell proliferation characteristic of human cancer. ${ }^{5}$ Although carcinogenesis is a multistep genetic process, restoration of p53 function has been shown to stabilise the malignant phenotype of neoplastic cells. ${ }^{67}$ Overexpression of wild type p53 by plasmid has been shown to be capable of driving p53 mutated gastric cancer cells into growth arrest and/or apoptosis. ${ }^{8}$

Recently, the adenoviral vector has emerged as a leading candidate for in vivo gene delivery, because of its broad host range, high viral titre, and high efficiency of gene transfer. ${ }^{9}{ }^{10}$ In contrast with the retrovirus, it is capable of transferring genes to a wide range of host cells including non-proliferating cells, which is preferable because of the heterogeneity of cell cycling within the tumour microenvironment.

To test the potential of $\mathrm{p} 53$ gene therapy for human gastric cancer, the effects of adenovirus mediated transfer of wild type p53 on in vitro and in vivo human gastric cancer cell lines were examined.

\section{Methods}

TUMOUR CELL LINES

Human gastric cancer cell lines (MKN1, MKN7, MKN28, and MKN45) and human embryonic kidney cell line 293 were obtained from the American Type Culture Collection. The TMK-1 gastric cancer cell line was kindly provided by Dr E Tahara (Hiroshima University, Japan). MKN1 cells were cultured in minimal essential medium supplemented with $10 \%$ fetal bovine serum. MKN7, MKN28, MKN45, and TMK-1 cells were cultured in RPMI 1640 medium supplemented with $10 \%$ fetal bovine serum. 293 Cells were cultured in Dulbecco's modified Eagle's medium containing $5 \%$ fetal bovine serum and $2 \mathrm{mM}$ glutamate.

Abbreviations used in this paper: moi, multiplicity of infection; X-gal, 5-bromo-4-chloro-3-indolyl- $\beta$-Dgalactopyranoside; MTT, 3-(4,5-dimethylthiazol-2yl)-2,5-diphenyltetrazolium bromide. 
Table 1 Adenovirus mediated gene transfer efficiency and growth inhibitory effect of AdCAp53 on human gastric cancer cell lines

\begin{tabular}{lllll}
\hline Cell line & $p 53$ status & $\beta$-gal & $E D_{50}$ AdCAlacZ & $E D_{50}$ AdCAp53 \\
\hline MKN1 & 143 Val to Ala & $92(7)$ & $>300$ & $2.5(0.2)$ \\
MKN7 & 278 Pro to Ser & $91(4)$ & $>300$ & $10.7(3.5)$ \\
MKN28 & 251 Ile to Leu & $92(5)$ & $>300$ & $99.0(24)$ \\
TMK-1 & 173 Val to Met & $90(2)$ & $>300$ & $90.0(17)$ \\
MKN45 & Wild type & $95(3)$ & $>300$ & $>300$ \\
\hline
\end{tabular}

Results are expressed as mean (SD) from three independent experiments.

p53 status was detected by DNA sequencing. ${ }^{22} \beta$-gal is the percentage of $\mathrm{X}$-gal positive cells 24 hours after AdCAlacZ infection at a multiplicity of infection (moi) of $30 . \mathrm{ED}_{50}$, moi required for a $50 \%$ reduction in viability of untreated cells.

RECOMBINANT ADENOVIRUS VECTORS

Recombinant replication deficient adenovirus vector AdCAp53 was constructed by a homologous recombination between an expression cosmid and the parental virus genome lacking the viral sequence regions E1A, E1B, and E3 described previously. ${ }^{11-13}$ AdCAp53 expresses human wild type p53 cDNA by a CAG promoter, which is composed of a cytomegalovirus immediate early enhancer and a modified chicken $\beta$-actin promoter. ${ }^{14}$ Preparation of the recombinant adenovirus AdCAlacZ (the lac $Z$ gene is driven by a CAG promoter) has been described previously. ${ }^{11}$ Adenoviruses were isolated from a single plaque and expanded in 293 cells, after which the resultant viral solutions were stored at $-80^{\circ} \mathrm{C}$. The virus titre was determined by plaque assay on 293 cells. ${ }^{11-13}$

$\beta$-GALACTOSIDASE EXPRESSION

Gastric cancer cells were plated in six-well culture plates (Corning, Acton, Massachusetts, USA) at a density of $5 \times 10^{5}$ cells/well and cultured overnight. Cells were infected with AdCAlacZ at a multiplicity of infection (moi) ranging from 1 to 100 . After 24 hours, 5-bromo-4-chloro-3-indolyl- $\beta$-D-galactopyranoside (X-gal) was used as a substrate to evaluate $\beta$-D-galactosidase expression, which is revealed by a blue precipitate in the cell. ${ }^{12}{ }^{13}$ Results are represented as mean (SD).

\section{ASSAY OF CELL GROWTH}

Cells were plated at a density of $5 \times 10^{3}$ cells $/ 100 \mu \mathrm{l}$ in 96-well plates (Corning), and were infected with either AdCAlacZ or AdCAp53 at various moi 24 hours later. Five days after adenoviral infection, cell growth was assessed by 3-(4,5-dimethylthiazol-2-yl)-2,5diphenyltetrazolium bromide (MTT) assay. ${ }^{13}$ Cell viability is proportional to the absorbance at the test wavelength $(570 \mathrm{~nm})$ from which the reference wavelength $(620 \mathrm{~nm})$ is subtracted. The moi of adenovirus required for a $50 \%$ reduction in viability of untreated controls was calculated by a curve fitting parameter. The results are presented as mean (SD) from three independent experiments.

WESTERN BLOT ANALYSIS

Cells in a $10 \mathrm{~cm}$ tissue culture dish (Corning) were infected with either AdCAlacZ or AdCAp53 at moi 30. At 24 hours after infection, total cell lysates were prepared by sonicating the cells in TNE buffer $(10 \mathrm{mM}$ Tris/ $\mathrm{HCl}(\mathrm{pH}$ 7.8), $1 \%$ Nonidet P40, $150 \mathrm{mM} \mathrm{NaCl}, 1 \mathrm{mM}$ EDTA, $10 \mu \mathrm{g} / \mathrm{ml}$ aprotinin). Protein $(20 \mu \mathrm{g})$ from the samples was subjected to sodium dodecyl sulphate $10 \%$ polyacrylamide gel electrophoresis and transferred to nitrocellulose membrane. The membrane was blocked with blocking buffer ( $5 \%$ non-fat dry milk and $0.2 \%$ Tween 20 in phosphate buffered saline) and probed with a mouse monoclonal anti-human p53 antibody, PAb1801 (Oncogene Research, Cambridge, Massachusetts, USA), followed by a goat anti-mouse IgG conjugated with horseradish peroxidase (Amersham, Arlington Heights, Illinois, USA). Reactive protein was detected by chemiluminescence (ECL kit; Amersham). The membrane was processed and developed according to the manufacturer's suggestions.

\section{DETECTION OF APOPTOSIS}

Flow cytometry

Detection of apoptotic cells by flow cytometry was performed as described previously. ${ }^{15}$ About $1 \times 10^{6}$ cells in $10 \mathrm{~cm}$ culture dishes were infected with AdCAlacZ or AdCAp53 at moi 30 for 24 hours and fixed in $70 \%$ ethanol/ phosphate buffered saline. After RNase treatment, cells were stained with $50 \mu \mathrm{g} / \mathrm{ml}$ propidium iodide (Molecular Probes, Eugene, Oregon, USA) in phosphate buffered saline. Flow cytometry was performed on a FACScan flow cytometer (Becton Dickinson, San Jose,
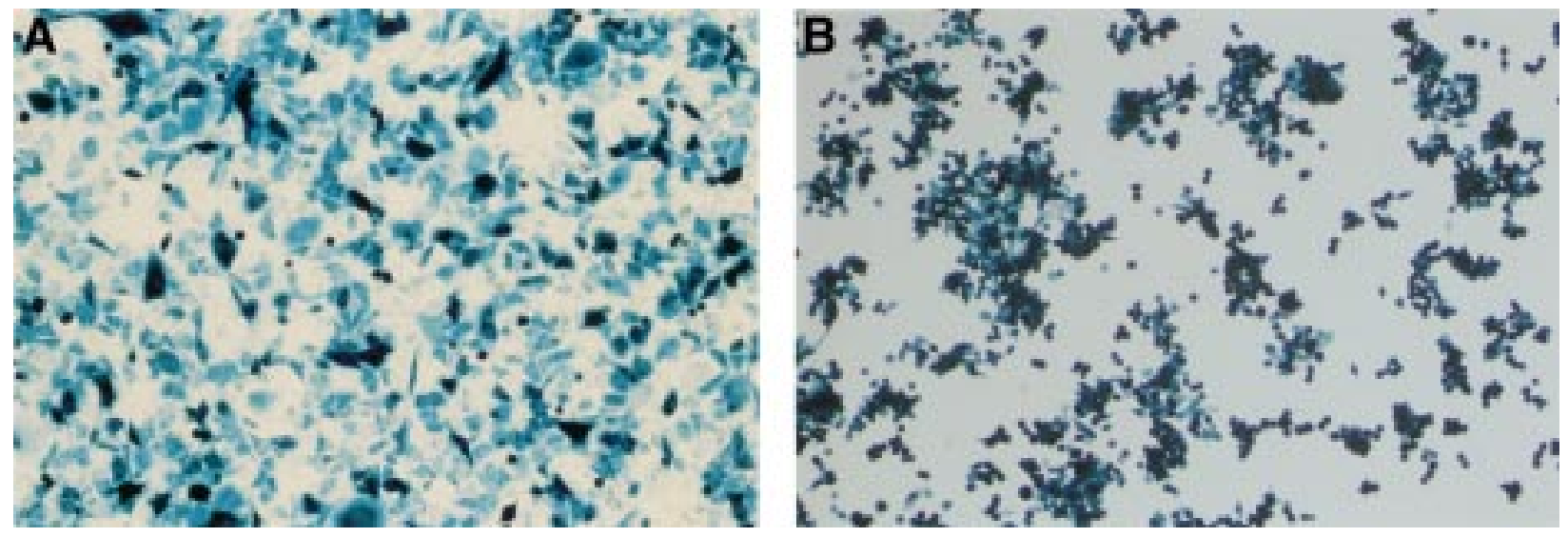

Figure 1 Transduction efficiency of adenovirus in gastric cancer cells. MKN1 (A) and MKN45 (B) cells were plated on six-well plates and infected with AdCAlacZ at a multiplicity of infection of 30. After 24 hours, cells were fixed and stained with 5-bromo-4-chloro-3-indolyl- $\beta$-D-galactopyranoside to demonstrate lac $Z$ gene expression. Original magnification $\times 100$. 

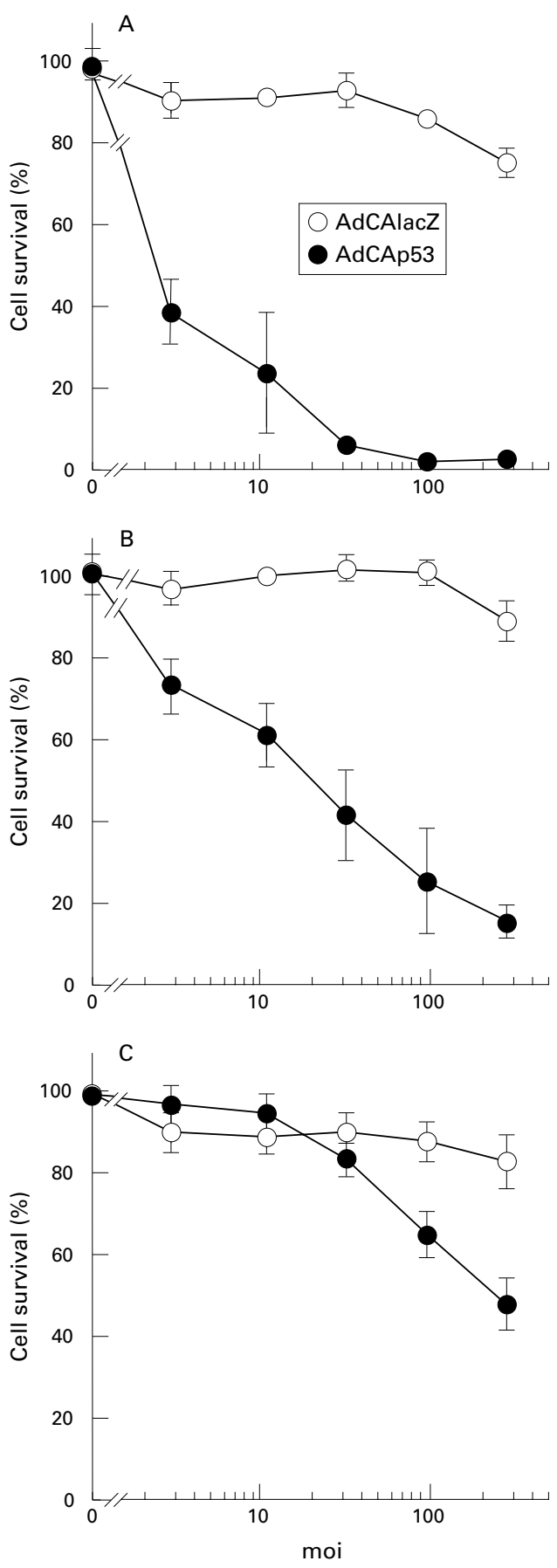

Figure 2 Effect of AdCAp53 on gastric cancer cell viability. Gastric cancer cells, $M K N 1$ ( $A$ ), MKN7 (B), and MKN45 (C), were infected with either AdCAlacZ or AdCAp53 at a multiplicity of infection (moi) ranging from 1 to 300. Five days after infection, effects on cell viability were measured by the 3-(4,5-dimethylthiazol-2-yl)2,5-diphenyltetrazolium bromide assay. Data are expressed as a percentage (mean (SD)) of the absorbance $\left(A_{570}-\right.$ $\left.A_{620}\right)$ of untreated cells.

California, USA), and the resulting DNA histograms of cell number versus integrated red fluorescence were analysed with Lysis II software (Becton Dickinson).

Detection of DNA laddering

About $5 \times 10^{5}$ cells in $10 \mathrm{~cm}$ culture dishes were treated with AdCAlacZ or AdCAp53 at moi 30. At 24 hours after infection, cells were har- vested, washed in phosphate buffered saline and lysed in $40 \mu \mathrm{l}$ lysis buffer containing 200 $\mathrm{mM} \mathrm{Na}_{2} \mathrm{HPO}_{4}$ and $4 \mathrm{mM}$ citric acid. Samples were centrifuged and the supernatants were incubated with $3 \mu \mathrm{l} 0.25 \%$ Nonidet P40 and $3 \mu \mathrm{l}$ DNase-free RNase $(10 \mathrm{mg} / \mathrm{ml})$ followed by $10 \mathrm{mg} / \mathrm{ml}$ proteinase $\mathrm{K}$ digestion. Aliquots of $10 \mu \mathrm{l}$ from $50 \mu \mathrm{l}$ DNA solution were electrophoresed on a $2.5 \%$ agarose gel in the presence of $0.5 \mu \mathrm{g} / \mathrm{ml}$ ethidium bromide.

ADENOVIRUS TREATMENT IN VIVO

$\mathrm{Balb} / \mathrm{c}$ athymic nude mice at six to eight weeks old (Clea Japan Inc, Tokyo, Japan) were used. Animal experiments were performed in accordance with the institutional guidelines of the university committee on the use and care of animals. Mice were inoculated with $2 \times 10^{6}$ MKN1 cells or MKN45 cells into the flank, and tumours were allowed to grow to $4 \mathrm{~mm}$ in diameter. On the previous day, MKN1 inoculated mice were given $500 \mu \mathrm{g}$ anti-asialo GM1 antibody in the tail vein. They were divided into three groups according to treatment schedule: AdCAp53 injection ( $n=6)$; AdCAlacZ injection $(n=6)$; and injection of control vehicle (Dulbecco's modified Eagle's medium $/ 5 \%$ fetal bovine serum) ( $\mathrm{n}=6$ ). Adenovirus vectors $\left(1 \times 10^{7}\right.$ plaque forming units $/ 50 \mu \mathrm{l})$ were directly injected into the foci centre at days 8,10 , and 12 . Tumour length and width were measured with calipers over a period of five weeks. Tumour volume was calculated from (length $\times$ width $\left.^{2}\right) / 2$ and presented as mean (SD) $\mathrm{mm}^{3}$. Statistical analysis was performed by the Mann-Whitney $\mathrm{U}$ test and StatView 4.11 software (Abacus Concepts Inc, Berkeley, California, USA). $\mathrm{p}<0.05$ was considered statistically significant.

\section{Results}

ADENOVIRUS MEDIATED GENE TRANSFER IN

GASTRIC CANCER CELLS

The efficiency of adenovirus gene transfer into gastric cancer cell lines was assessed as the percentage of X-gal staining positive cells 24 hours after infection with AdCAlacZ. Table 1 summarises the extent of $\beta$-gal expression of the human gastric cancer cell lines. AdCAlacZ could efficiently transfer the lac $Z$ gene into these cell lines, and X-gal-positive cells at moi 30 were $92 \%$ of $\mathrm{MKN} 1$ (fig $1 \mathrm{~A}$ ), $91 \%$ of MKN7, $92 \%$ of MKN28, $90 \%$ of TMK-1, and $95 \%$ of MKN45 (fig 1B) cells respectively.

INHIBITION OF CELL GROWTH IN VITRO

Degree of growth inhibition was measured as the dose required for a $50 \%$ inhibition in the MTT assay. In the p53-mutated gastric cancer cell lines, the inhibition of cell growth by AdCAp53 correlated with adenoviral transduction efficiency except for in MKN28 cells and TMK-1 cells (table 1). Further, infection with AdCAp53 at moi 30 significantly inhibited the growth of MKN1 (fig 2A) and MKN7 (fig 2B) cells, which are susceptible to adenovirus infection. Despite the efficient gene transfer by AdCAlacZ of MKN28 and TMK-1 cells, the p53-specific inhibition of cell growth by AdCAp53 was minimal in these cell lines (table 


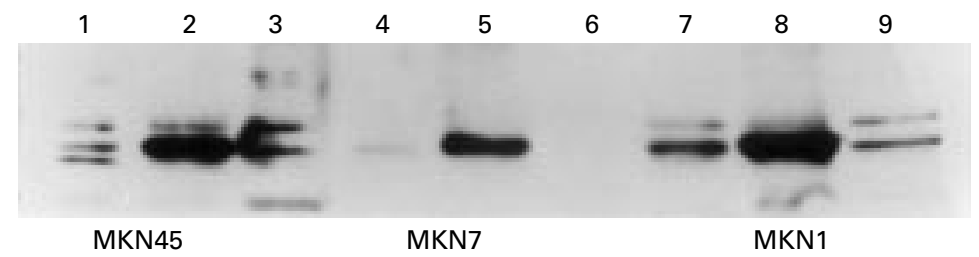

Figure 3 Adenovirus mediated p53 expression in human gastric cancer cells. MKN45, MKN7, and MKN1 cells were left untreated (lanes 1, 4, and 7) or were infected with AdCAp53 (lanes 2, 5, and 8) or AdCAlacZ (lanes 3, 6, and 9) at a multiplicity of infection of 30. Western blot analysis of cell lysates was performed with the p53-specific antibody.

1). In the gastric cancer cells with wild type $\mathrm{p} 53$ gene, MKN45 cells, the cell growth was not inhibited by AdCAp53 infection (fig 2C).

p53 EXPRESSION IN AdCAp53 INFECTED CELLS According to the efficiency of gene transfer by AdCAlacZ on gastric cancer cell lines, the cells were infected with AdCAp53 at moi 30. The expression of $\mathrm{p} 53$ protein in the cells was

A

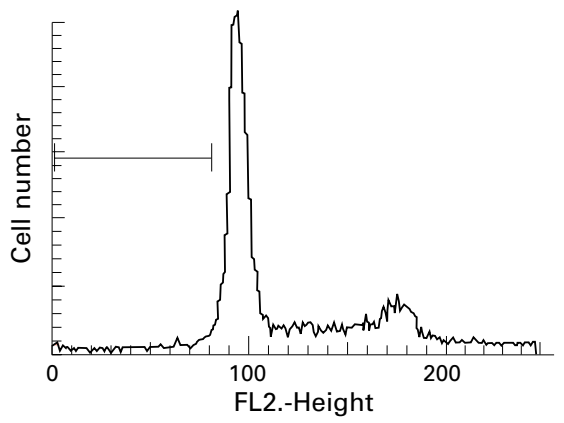

B

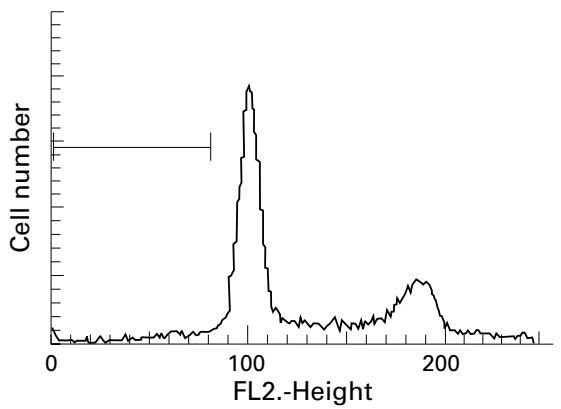

C

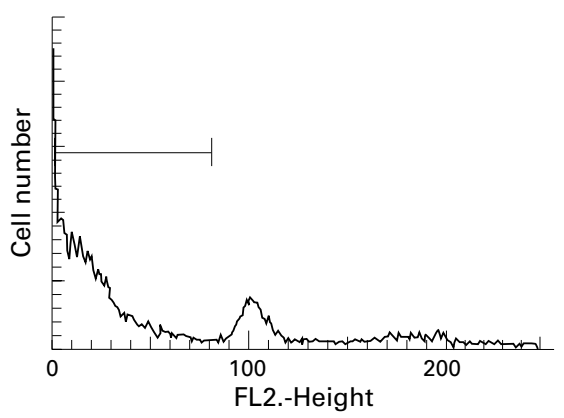

DNA contents detected by western blot analysis using a specific anti-p53 antibody, PAb1801, which recognises both wild type and mutant p53 protein. A p53 band recognised by PAb1801 was observed in cellular extracts isolated from all samples. Samples isolated from mock infected gastric cancer cells exhibited very low levels of p 53 protein (fig 3 , lanes 1, 4, and 7). A high level of expression of p53 protein was achieved in MKN45, MKN7, and MKN1 cells infected with AdCAp53 (fig 3, lanes 2,5 , and 8 ). In contrast, the levels of p53 expression remained unchanged in the cells infected with AdCAlacZ (fig 3, lanes 3, 6, and 9). These results indicate that the exogenous p53 gene introduced by AdCAp53 is efficiently translated into $\mathrm{p} 53$ protein.

\section{INDUCTION OF APOPTOSIS}

A flow cytometric analysis was performed on propidium iodide stained cancer cells infected

D

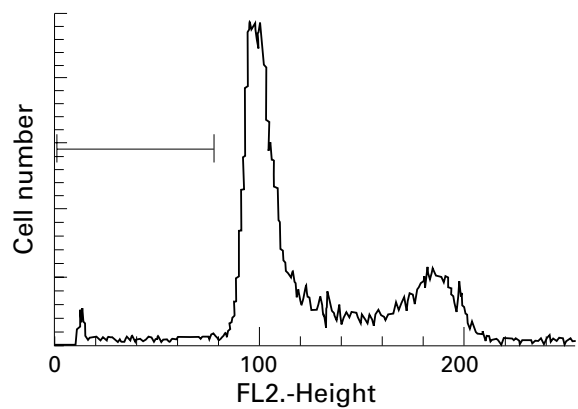

E

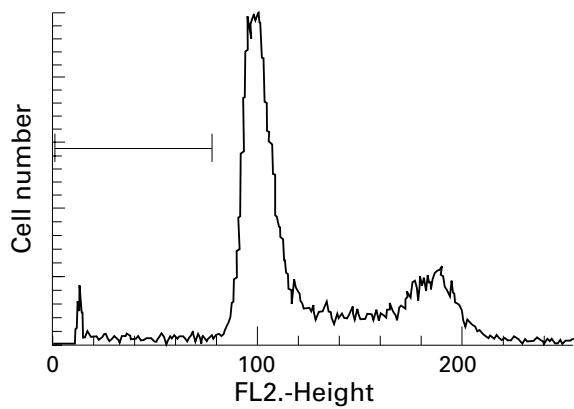

$\mathrm{F}$

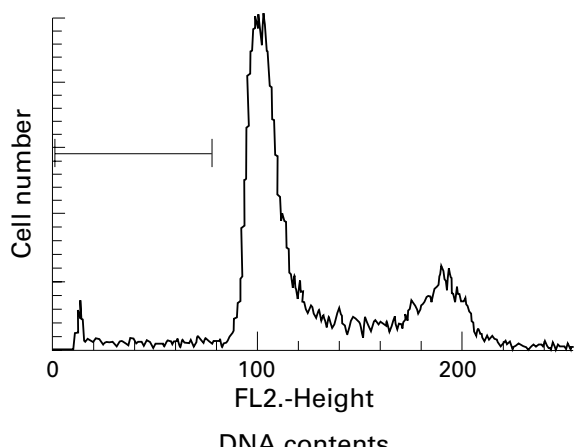

Figure 4 Detection of apoptotic cells by flow cytometry. About $1 \times 10^{6}$ MKN1 and MKN45 cells were left untreated (A, $D)$ or were treated with AdCAlacZ $(B, E)$ or AdCAp53 $(C, F)$ at a multiplicity of infection of 30 . After 24 hours, cells were centrifuged and fixed in $70 \%$ ethanol. Samples were then treated with RNase, stained with propium iodide, and analysed by flow cytometry. Untreated control (A) or AdCAlacZ treated (B) MKN1 cells showed little sub-G1 peak. AdCAp53 treated MKN1 cells showed a significant sub-G1 peak indicative of apoptosis $(C)$. The flow cytometry histograms for untreated control, AdCAlacZ treated, and AdCAp53 treated MKN45 cells show no significant hypodiploid fraction $(D, E, F)$. 


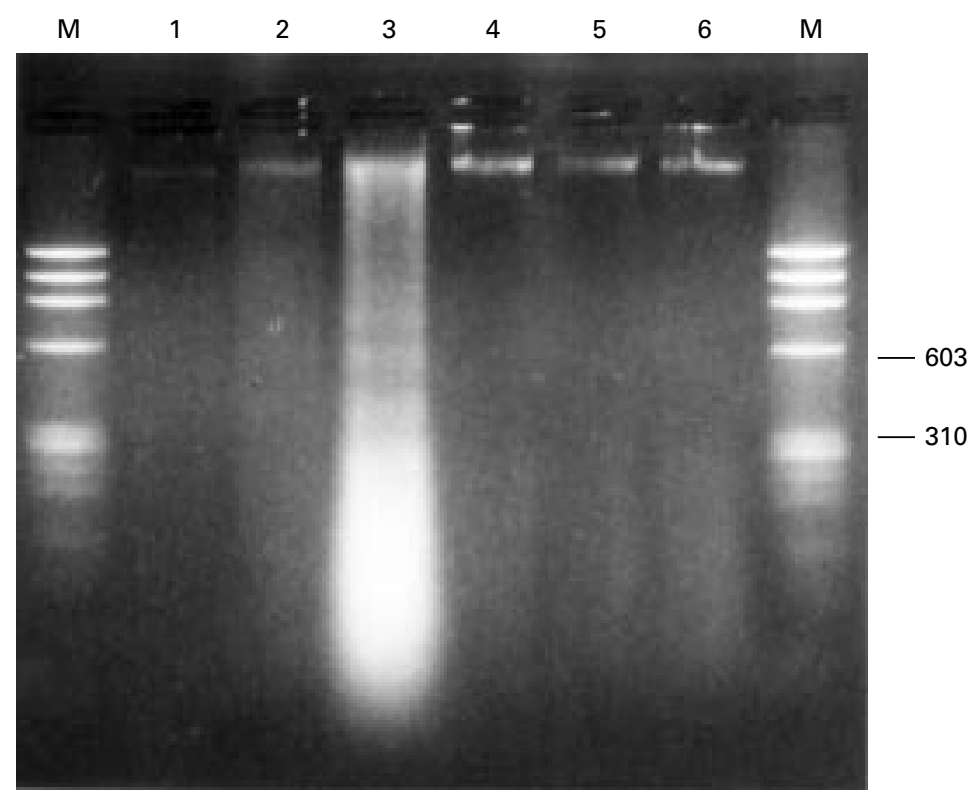

Figure 5 DNA fragmentation induced by AdCAp53 infection. MKN1 and MKN45 cells were untreated (lanes 1 and 4) or treated with AdCAlacZ (lanes 2 and 5) or AdCAp53 (lanes 3 and 6) at a multiplicity of infection of 30 . The positions of molecular mass markers (lane $M$ ) are indicated on the right (base). DNA was prepared from $5 \times 10^{5}$ cells as described in the Methods section; $10 \mu \mathrm{l}$ aliquots from $50 \mu \mathrm{l}$ DNA solution were separated on a $2.5 \%$ agarose gel in the presence of $0.5 \mu \mathrm{g} / \mathrm{ml}$ ethidium bromide.

with adenovirus vectors to analyse occurrence of apoptosis. Owing to DNA fragmentation, apoptotic cells have lower DNA content and display a sub-G1 peak in the flow cytometry histogram. As shown in fig 4C, a sub-G1 peak was detected in AdCAp53 treated MKN1 cells, whereas the flow cytometry histogram for both untreated and AdCAlacZ treated $\mathrm{MKN1}$ cells revealed little sub-G1 fraction (fig. 4A, B). The percentage of cells in the sub-G1 peak was $1.2 \%$ of untreated, $2.6 \%$ of AdCAlacZ treated, and $62.9 \%$ of AdCAp53 treated MKN1 cells respectively. The flow cytometry histogram for untreated, AdCAlacZ treated, and AdCAp53 treated MKN45 cells showed no significant hypodiploid fraction (less than 3\%) (fig $4 \mathrm{D}, \mathrm{E}$, F).

DNA of MKN1 cells infected with AdCAp53 showed a typical DNA ladder (fig 5, lane 3). A
DNA ladder was not detected in untreated cells (fig 5, lanes 1 and 4), in cells treated with AdCAlacZ (fig 5, lanes 2 and 5), or in MKN45 cells treated with AdCAp53 (fig 5, lane 6).

These data suggest apoptosis as the mechanism of gastric cancer cell death after AdCAp53 treatment.

\section{INHIBITION OF TUMOUR GROWTH IN VIVO}

We assessed the efficacy of the AdCAp53 treatment for subcutaneous tumours of gastric cancer. Recombinant adenoviruses (AdCAp53, AdCAlacZ), or control vehicle were injected directly into the established subcutaneous MKN1 and MKN45 tumours. Figure 6A shows that the growth of MKN1 tumours was significantly inhibited by treatment with AdCAp53 compared with treatment with AdCAlac $Z$ or control vehicle $(p<0.001)$. Average tumour volumes on day 30 were 43 (6) $\mathrm{mm}^{3}$ for the AdCAp53 treated mice, 332 (25) $\mathrm{mm}^{3}$ for those treated with control vehicle, and 286 (33) $\mathrm{mm}^{3}$ for the AdCAlacZ treated mice. Mice inoculated with MKN45 cells developed large tumours of more than $400 \mathrm{~mm}^{3}$ on day 24 and there was no significant difference in tumour growth among the three groups. After day 29, tumours treated with AdCAlacZ showed a slightly decreased growth curve. However, the difference between the growth of untreated and AdCAp53 treated tumours was not statistically significant (fig 6B). Data from these animal experiments show that adenovirus mediated p53 gene transfer is capable of suppressing the growth of established tumours derived from human p53 mutant gastric cancer cells.

\section{Discussion}

The p53 protein is highly conserved throughout evolution and is expressed in all tissues of the human body. Wild type p53 has been shown to be involved in transcriptional regulation, cell cycle arrest at the G1/S check point, and induction of apoptosis. ${ }^{4}$ Furthermore, it is capable of suppressing cell transformation and neoplastic cell growth. ${ }^{6}$ p53 gene replacement therapy in cells with an absent or mutated p53 gene is an appealing concept, and data from in
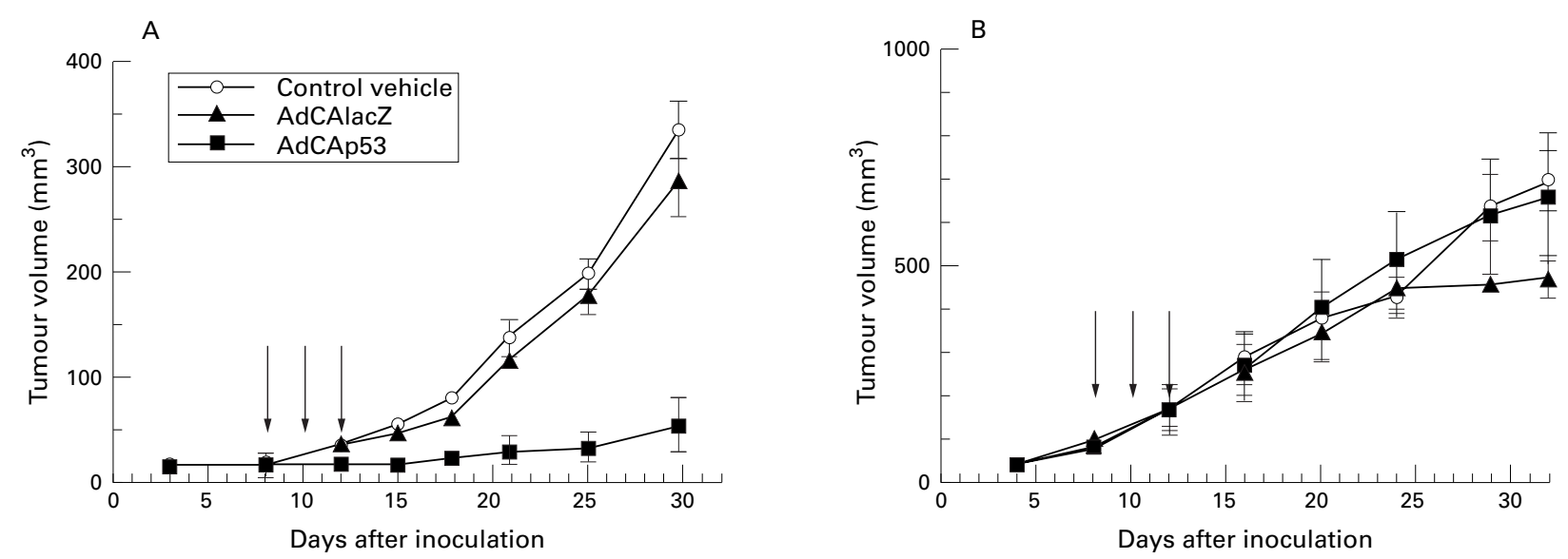

Figure 6 Effect of AdCAp53 on established tumours. After reaching a size of $4 \mathrm{~mm}$ in diameter, tumours were treated with three injections (arrows) on days 8,10, and 12 of control vehicle or adenovirus (total dose $3 \times 10^{7}$ plaque forming units). Tumour volume was plotted for each treatment animal group $(n=6)$ against days after inoculation (mean (SE)). (A) Over the 30 day experimental period, MKN1 tumour growth was significantly suppressed in AdCAp53 treated mice in comparison with those treated with control vehicle or AdCAlacZ ( $p<0.001)$. (B) In MKN45 tumour growth, there was no significant difference between untreated and AdCAp53 treated animals. 
vitro and in vivo studies have been encouraging. ${ }^{16-18}$ p53 gene therapy may also be an effective strategy for increasing the sensitivity of cancer cells to conventional anticancer treatment such as chemotherapy and radiation therapy. ${ }^{16}$ It is reported that transient expression of wild type p53 can inhibit the growth of human gastric cancer cell lines. ${ }^{8}$

In this study, the effects of AdCAp53 on human gastric cancer cell lines were examined both in vitro and in vivo. Firstly, the extent of the adenovirus mediated gene transfer was evaluated by measuring $\beta$-gal expression of the cells infected with recombinant adenoviruses encoding the lac $Z$ gene. AdCAlacZ vectors could transfer the $l a c Z$ gene into more than $90 \%$ of five gastric cancer cell lines examined at moi 30. Further, AdCAp53 could successfully deliver and express p 53 protein into both wild type and mutated p53 gastric cancer cell lines. These data show the capability of adenovirus to transfer exogenous genes efficiently into gastric cancer cell lines. Secondly, the growth inhibitory effect of AdCAp53 was then examined. In p53 mutated gastric cancer cells, the growth inhibition by infection with AdCAp53 correlated with both transduction efficiency and adenoviral dose except in MKN28 and TMK-1 cells. Moreover, flow cytometric analysis and DNA fragmentation suggested that the cytotoxic effect of reintroduced p53 was the result of apoptosis.

There was some discrepancy between the degree of adenovirus gene transduction efficiency and the p53 mediated growth inhibition in MKN28 and TMK-1 cells. This discrepancy may be the result of suppression of the wild type $\mathrm{p} 53$ protein by the increased mutated p53 protein. This seems unlikely as Harris et $a l^{17}$ reported that high levels of endogenous mutated p 53 protein did not abrogate the antiproliferative effect of wild type p53 protein. However, as the p53 mutational status of the MKN28 and TMK-1 cells differed from that of p53 mutated cancer cell lines examined by Harris et al, this possibility cannot be completely excluded. Another possible explanation is that molecules in the p53 dependent apoptosis pathway are altered. Reintroduction of wild type p53 does not cause apoptosis if downstream molecules of the pathway have no or insufficient function. Although little is known about the p53 dependent apoptosis pathway, recent studies have implicated the Bax protein in this process. ${ }^{19}$ Further investigation of these cancer cells is required to clarify the underlying mechanisms of the insufficient inhibition of cell growth by $\mathrm{p} 53$ reintroduction.

Encouraging results were also obtained in studies using nude mice. Local AdCAp53 treatment by intra- or peri-tumoral injection led to a significant inhibition of tumour growth consistent with the in vitro effect of AdCAp53 on human gastric cancer cells. In AdCAp53 treated animals, two of six MKN1 tumours regrew 20 days after adenoviral injection. Several studies revealed that intratumoral injection of recombinant adenoviruses results in nonuniform transgene expression in subcutaneous tumours. $^{2021}$ This limitation may provide a likely explanation for the eventual regrowth of
AdCAp53 treated tumours. Regional targeting of the vascular supply of malignancies, such as hepatic artery administration to liver tumours, may significantly improve cell transduction in vivo. ${ }^{18}$

In summary, this study was designed to evaluate the efficiency and potential applicability of in vivo p53 gene therapy in an animal model of human gastric cancer. Our findings of significant tumour reduction in p53-altered human gastric cancer by adenovirus mediated p53 gene transfer seem to provide a sound basis for future clinical trials of gastric cancer gene therapy.

This work was supported in part by grants from the Ministry of Health and Welfare and the Ministry of Education, Culture and Science, Japan. We thank $M$ Tsubouchi and $\mathrm{H}$ Matsuda for technical assistance and S Shimisu for reading the manuscript.

1 Wanebo HJ, Kennedy BJ, Chmiel J, et al. Cancer of the stomach. A patient care study by the American College of Surgeons. Ann Surg 1993;218:583-92.

2 Thompson GB, van Heerden JA, Sarr MG. Adenocarcinoma of the stomach: are we making progress? Lancet noma of the stomac

3 Weinberg RA. Tumor suppressor genes. Science 1991;254: 1138-46.

4 Levine AJ. p53, the cellular gatekeeper for growth and division. Cell 1997;88:323-31.

5 Tahara E, Semba S, Tahara H. Molecular biological observations in gastric cancer. Semin Oncol 1996;23:307-15.

6 Baker SJ, Markowitz S, Fearon ER, et al. Suppression of human colorectal carcinoma cell growth by wild-type $\mathrm{p} 53$. Science 1990;249:912-15.

7 Mercer WE, Shields MT, Amin M, et al. Negative growth regulation in a glioblastoma tumor cell line that conditionally expresses human wild-type p53. Proc Natl Acad Sci USA 1990;87:6166-70.

8 Matozaki T, Sakamoto C, Suzuki T, et al. p53 gene mutations in human gastric cancer: wild-type p 53 but not mutant p 53 suppresses growth of human gastric cancer cells. Cancer Res 1992;52:4335-41.

9 Berkner KL. Expression of heterologous sequences in adenoviral vectors. Curr Top Microbiol Immunol 1992;158: 39-66.

10 Marcel T, Grausz JD. The TMC Worldwide Gene Therapy Enrollment Report, end 1996. Hum Gene Ther 1997;8: 775-800.

11 Miyake S, Makimura M, Kanegae Y, et al. Efficient generation of recombinant adenoviruses using adenovirus DNAterminal protein complex and a cosmid bearing the full-length virus genome. Proc Natl Acad Sci USA 1996;93: 1320-4.

12 Lan K-H, Kanai F, Shiratori Y, et al. Tumor-specific gene expression in carcinoembryonic antigen-producing gastric
cancer cells using adenovirus vectors. Gastroenterology cancer cells using

13 Kanai F, Lan K-H, Shiratori Y, et al. In vivo gene therapy for alpha-fetoprotein-producing hepatocellular carcinoma by adenovirus-mediated transfer of cytosine deaminase gene. Cancer Res 1997;57:461-5

14 Niwa H, Yamamura K, Miyazaki J. Efficient selection for high-expression transfectants with a novel eukaryotic vector. Gene 1991;108:193-9.

15 Darzynkiewicz Z, Bruno S, Del Bino G, et al. Features of apoptotic cells measured by flow cytometry. Cytometry 1992;13:795-808.

16 Fujiwara T, Grimm EA, Mukhopadhyay T, et al. Induction of chemosensitivity in human lung cancer cells in vivo by adenovirus-mediated transfer of the wild-type p53 gene. Cancer Res 1994;54:2287-91.

17 Harris MP, Sutipto S, Wills KN, et al. Adenovirus-mediated p53 gene transfer inhibits growth of human tumor cells p53 gene transfer inhibits growth of human tumor cells 3:121-30.

18 Bookstein R, Demers W, Gregory R, et al. p53 gene therapy in vivo of herpatocellular and liver metastatic colorectal cancer. Semin Oncol 1996;23:66-77.

19 Miyashita T, Reed JC. Tumor suppressor p53 is a direct transcriptional activator of the human bax gene. Cell 1995; 80:293-9.

20 Hirschowitz EA, Ohwada A, Pascal WR, et al. In vivo adenovirus-mediated gene transfer of the Escherichia coli cytosine deaminase gene to human colon carcinomaderived tumors induces chemosensitivity to 5-fluorocytosine. Hum Gene Ther 1995;6:1055-63.

21 Wills KN, Huang W-M, Harris MP, et al. Gene therapy for hepatocellular carcinoma: chemosensitivity conferred by adenovirus-mediated transfer of the HSV-1 thymidine adenovirus-mediated transfer of the HSV-1

22 Yanagihara $\mathrm{K}$, Numoto $\mathrm{M}$, Tauchi $\mathrm{H}$, et al. Genetic status of p53 and induction of apoptosis by radiation or isoflavones in human gastric carcinoma cell lines. International fournal of Oncology 1996;9:95-102. 